\title{
pT classification, grade, and vascular invasion as prognostic indicators in urothelial carcinoma of the upper urinary tract
}

\author{
Cord Langner ${ }^{1}$, Georg Hutterer ${ }^{2}$, Thomas Chromecki ${ }^{2}$, Ingrid Winkelmayer ${ }^{1}$, Peter Rehak $^{3}$ \\ and Richard Zigeuner ${ }^{2}$
}

${ }^{1}$ Institute of Pathology, Medical University Graz, Graz, Austria; ${ }^{2}$ Department of Urology, Medical University Graz, Graz, Austria and ${ }^{3}$ Division of Biomedical Engineering \& Computing, Department of Surgery, Medical University Graz, Graz, Austria

\begin{abstract}
Clinicopathologic features predictive of patient outcome in upper urinary tract urothelial carcinoma are not well defined. The aim of this study was to assess the role of pT classification, tumor grade, and vascular invasion in predicting metastasis-free survival. A total of 190 consecutive invasive upper urinary tract urothelial cancers operated between 01/1984 and 12/2004 were re-evaluated with respect to pT classification, tumor grade (according to the three-tiered WHO 1973 and the recent two-tiered grading system following the WHO/ISUP consensus classification), as well as presence of lymph and/or blood vessel invasion. Prognostic impact was analyzed using the Kaplan-Meier method and the Log-Rank test. For multivariate testing, a Cox's proportional hazards regression model was used. pT1 was present in $81(43 \%)$, pT2 in $29(15 \%)$, pT3 in $73(38 \%)$, and pT4 in seven (4\%) cases. There were $12(6 \%)$ G1, $96(51 \%)$ G2, and $82(43 \%)$ G3 tumors or $84(44 \%)$ low-grade and 106 $(56 \%)$ high-grade tumors according to the two-tiered system. The presence of vascular invasion in $72 / 190(38 \%)$ tumors was associated with high pT classification $(P<0.001)$ and high tumor grade $(P<0.001)$. Disease progression occurred in $39 \%$ of patients, with 5- and 10 -year metastasis-free survival rates of 56 and $45 \%$, respectively. On univariate analysis, all investigated parameters showed prognostic significance. The negative influence of vascular invasion on patient outcome was strikingly strong in high pT classification and high-grade cancers. On multivariate analysis, pT classification $(P<0.001)$ and vascular invasion $(P<0.001)$ proved to be independent prognostic factors, whereas tumor grade according to the two-tiered system missed statistical significance $(P=0.06)$. In conclusion, $\mathrm{pT}$ classification and vascular invasion are independent prognostic factors with respect to metastasis-free survival and should be used to guide adjuvant therapy strategies in affected patients. Presence (or absence) of vascular invasion should be commented upon separately in the pathology report.
\end{abstract}

Modern Pathology (2006) 19, 272-279. doi:10.1038/modpathol.3800529

Keywords: urothelial carcinoma; upper urinary tract; tumor classification; tumor grade; vascular invasion; prognosis

A significant proportion of patients with upper urinary tract urothelial carcinoma (UC) eventually die of the disease due to unrecognized and untreated nodal or distant metastasis present at the time of surgery, indicating the importance of adequate adjuvant therapy strategies for patients at high risk of failure. Initial tumor stage and tumor grade have been documented as major prognostic factors. ${ }^{1-25}$ However, validity of most studies is

Correspondence: Dr C Langner, MD, Institute of Pathology, Medical University Graz, Auenbruggerplatz 25, A-8036 Graz, Austria.

E-mail: cord.langner@meduni-graz.at

Received 31 August 2005; revised 20 October 2005; accepted 21 October 2005 limited due to inclusion of noninvasive (pTa) cancer cases and/or small sample size. Thus, to the best of our knowledge, there are only two systematic studies involving more than 100 invasive cancer cases including both univariate and multivariate statistical analyses with respect to recurrence-free and disease-specific survival. ${ }^{12,24}$ Moreover, the assessment of tumor grade may show considerable observer variation, ${ }^{26}$ and the new two-tiered WHO grading system ${ }^{27,28}$ has only rarely been applied with respect to prognostic impact in upper urinary tract UC. ${ }^{22,24}$ Thus, other factors accurately predicting the biological potential of this malignancy are required to select candidates for adjuvant therapy. Vascular invasion has been identified as a promising 
additional marker. ${ }^{11,14,17,23,24,29}$ However, a systematic analysis of lymph and/or blood vessel invasion with respect to prognostic impact along different pT classifications and grades of both pelvic and ureteral UCs has not yet been performed.

Therefore, our study aimed to evaluate the prognostic influence of possible conventional variables, such as pT classification, tumor grade, tumor location, as well as lymph and/or blood vessel invasion, on metastasis-free survival in a large series of consecutive patients with upper urinary tract cancer.

\section{Materials and methods}

\section{Patient Selection and Tissues}

A total of 268 consecutive upper urinary tract UCs (169 pelvic and 99 ureteral tumors) from 239 consecutive patients (108 female and 131 male subjects) operated between 01/1984 and 12/2004 were retrieved from the archives of our institute. In a first step, all noninvasive cancers (41 tumors) and in a second all tumors from patients with multiple invasive cancers (37 tumors; since it is impossible to decide which of the UCs is responsible for disease progression) were excluded leading to a final sample of 190 UCs (134 pelvic and 56 ureteral tumors) from 190 patients (77 female and 113 male subjects) that were included in the investigation. Mean and median age of these patients was 70 and 71 (range 39-89) years, respectively. Patients either underwent nephroureterectomy with bladder-cuff resection $(n=130)$, nephrectomy alone $(n=40)$, or distal ureteral resection with reimplant $(n=20)$. Patients did not receive adjuvant therapy. Follow-up regimen included abdominal ultrasound and cystoscopy at 3-month intervals for the first 3 years, 6-month intervals for the subsequent 2 years, and yearly thereafter, as well as chest X-ray and computerized tomography at 6-month intervals for the first 3 years and yearly thereafter. In all, 54/190 (28\%) patients developed superficial bladder recurrence, which was not considered in the metastasis-free survival calculation since it had no impact on patient outcome in the study population (data not shown). All procedures were in accord with the ethical standards established by our institution. Informed consent is not required for retrospective analyses dealing with archival material obtained during routine medical treatment.

All tumor specimens had been examined according to the same protocol. The charts with the original macroscopic descriptions and the hematoxylin and eosin (H\&E)-stained slides from routinely formalin-fixed and paraffin-embedded material were systematically re-evaluated by one pathologist (CL), with special expertise in the field of uropathology, who was blinded to the regional lymph node status and the subsequent disease course. pT classifications were adjusted according to the UICC 2002 issue of the TNM system. ${ }^{30}$ In addition, pT3 tumors of the renal pelvis were subdivided regarding different patterns of tumor involvement as follows: (i) tumors invading renal parenchyma on a microscopic level only, (ii) tumors with macroscopic renal parenchyma invasion discerned from the gross appearance of the resection specimen, and (iii) tumors with spread into peripelvic fat. Grading was performed according to the three-tiered WHO guidelines published in $1973^{31}$ and the recent two-tiered WHO system ${ }^{27}$ for the grading of urothelial cancers which follows the WHO/ISUP consensus classification published in $1998 .^{28}$ In a last step, the slides were examined with respect to lymph vessel (L1) and/or blood vessel (V1) invasion. Only vessels with an unequivocal endothelial lining were considered true lymphatic vessels (Figure 1a). When carcinoma was present in vessels with a thick vascular wall and red blood cells in the lumen, this was considered blood vessel invasion (Figure 1b).
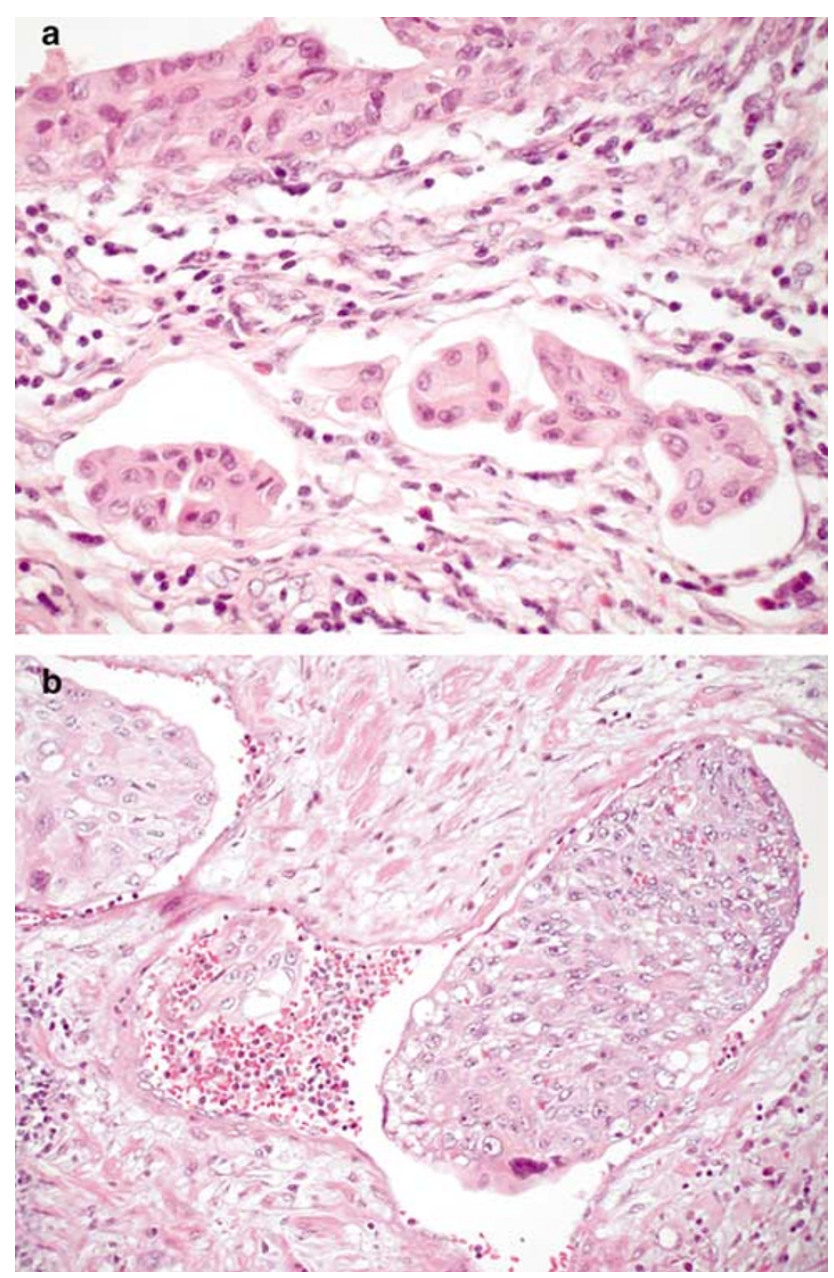

Figure 1 Urothelial carcinoma of the upper urinary tract with tumor invasion in lymphatic vessels (a) and blood vessels (b). 


\section{Statistical Analysis}

Associations between pT classification, tumor grade, and vascular invasion were analyzed using the Fisher's exact test. Metastasis-free survival was assessed by the Kaplan-Meier method and compared by the Log-Rank test. For multivariate testing, a Cox's proportional hazards regression model including patient age, gender, pT classification, tumor grade, vascular invasion, and tumor location (pelvic vs ureteral) was performed. All $P$-values were two-sided with significance at $P<0.05$.

\section{Results}

\section{Pathological Findings}

Overall, classification pT1 was present in 81 (43\%), pT2 in $29(15 \%)$, pT3 in $73(38 \%)$, and pT4 in seven $(4 \%)$ cases. With respect to the different subcategories among the 57 pelvic pT3 UCs, 13 (23\%) tumors showed microscopic infiltration of renal parenchyma only, whereas 33 (58\%) tumors infiltrated the renal parenchyma on a macroscopic level. A total of $28(49 \%)$ of the latter additionally invaded peripelvic fat. The remaining $11(19 \%)$ tumors showed fat invasion without evidence of renal parenchyma infiltration.

According to the WHO 1973 grading system, 12 (6\%) tumors were well differentiated (G1), 96 (51\%) moderately differentiated (G2), and $82(43 \%)$ poorly differentiated (G3). Regarding the recent two-tiered system, $84(44 \%)$ tumors were of low tumor grade and $106(56 \%)$ of high tumor grade. All former G1 tumors were included in the new low-grade and all former G3 tumors in the new high-grade category. With respect to former G2 tumors, $72(75 \%)$ were reclassified as low-grade cancers and $24(25 \%)$ tumors as high-grade cancers (Figure 2). Low tumor grade was associated with low tumor classification $(71 / 110(65 \%)$ pT1/pT2 vs 13/80 (16\%) pT3/pT4, $P<0.001)$, and high tumor grade was associated with high tumor classification $(39 / 110(35 \%)$ pT1/ pT2 vs $67 / 80(84 \%)$ pT3/pT4, $P<0.001)$.
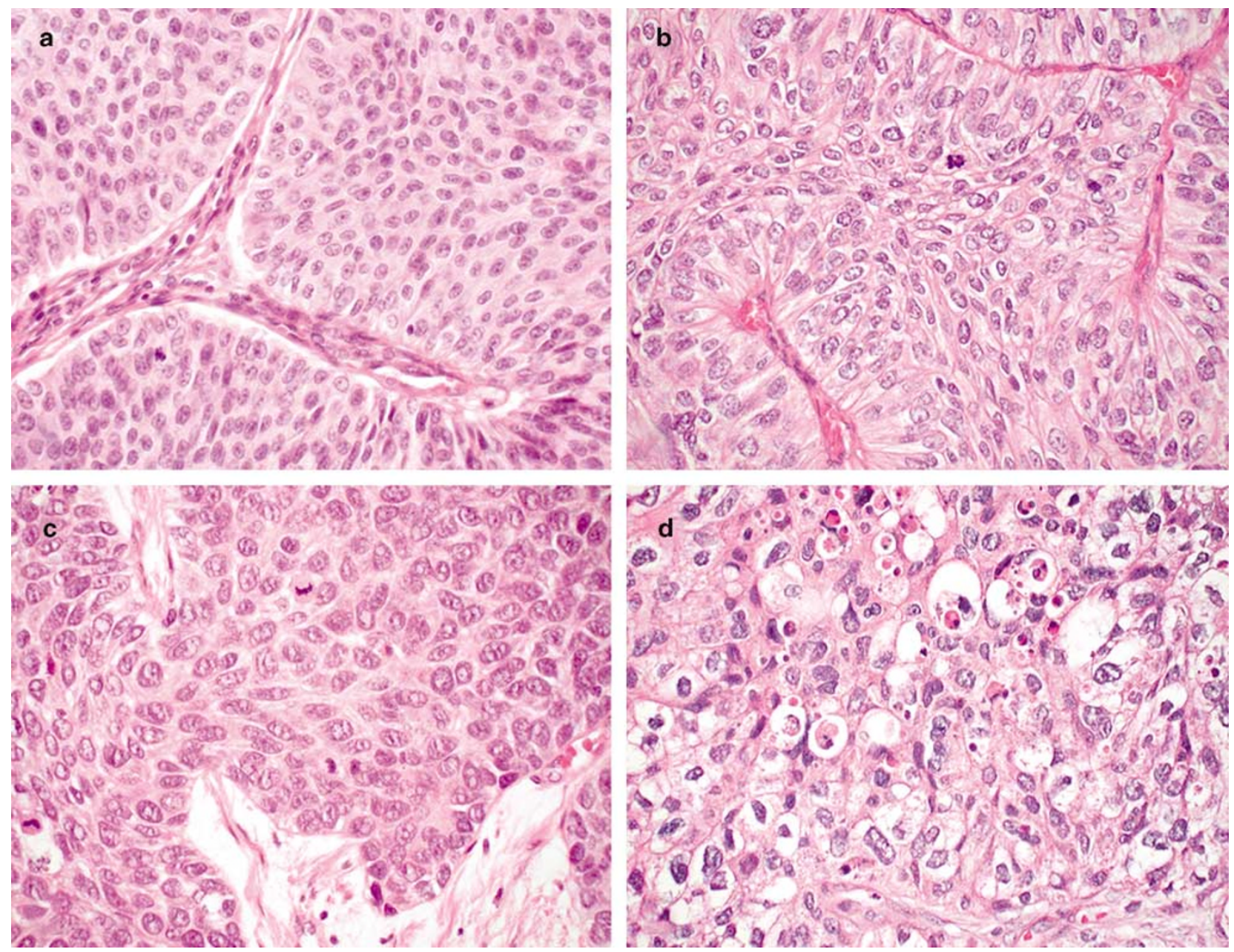

Figure 2 Urothelial carcinoma of the upper urinary tract. Tumor grades according the WHO 1973 grading system: well-differentiated cancer $(\mathrm{G} 1, \mathbf{a})$ and poorly differentiated cancer $(\mathrm{G} 3, \mathbf{d})$. Examples of former moderately differentiated cancer (G2) that were reclassified as either low-grade cancer (b) or high-grade cancer (c) owing to the recent two-tiered WHO grading system. 
Table 1 Presence of lymph vessel (L1) and blood vessel (V1) invasion in upper urinary tract urothelial carcinomas

\begin{tabular}{|c|c|c|c|c|c|c|}
\hline & \multicolumn{2}{|c|}{ All tumors $(\mathrm{n}=190)$} & \multicolumn{2}{|c|}{ Pelvic tumors $(\mathrm{n}=134)$} & \multicolumn{2}{|c|}{ Ureteral tumors $(\mathrm{n}=56)$} \\
\hline & $\mathrm{n}$ & $\%$ & $\mathrm{n}$ & $\%$ & $\mathrm{n}$ & $\%$ \\
\hline L0 and V0 & 118 & 62 & 81 & 60 & 37 & 66 \\
\hline L1 & 24 & 13 & 16 & 12 & 8 & 14 \\
\hline V1 & 12 & 6 & 9 & 7 & 3 & 5 \\
\hline L1 and V1 & 36 & 19 & 28 & 21 & 8 & 14 \\
\hline L1 and/or V1 & 72 & 38 & 53 & 40 & 19 & 34 \\
\hline pT1 & $10 / 81$ & 12 & $4 / 53$ & 8 & $6 / 28$ & 21 \\
\hline pT2 & $7 / 29$ & 24 & $4 / 17$ & 24 & $3 / 12$ & 25 \\
\hline pT3 & $48 / 73$ & 66 & $38 / 57$ & 67 & $10 / 16$ & 63 \\
\hline pT4 & $7 / 7$ & 100 & $7 / 7$ & 100 & - & - \\
\hline G1 & $0 / 12$ & 0 & $0 / 10$ & 0 & $0 / 2$ & 0 \\
\hline G2 & $19 / 96$ & 20 & $14 / 69$ & 20 & $5 / 27$ & 19 \\
\hline G3 & $53 / 82$ & 65 & $39 / 55$ & 71 & $14 / 27$ & 52 \\
\hline Low grade & $7 / 84$ & 8 & $5 / 60$ & 8 & $2 / 24$ & 8 \\
\hline High grade & $65 / 106$ & 61 & $48 / 74$ & 65 & $17 / 32$ & 53 \\
\hline
\end{tabular}

Overall vascular invasion (L1 and/or V1) related to tumor classification (pT1-pT4) and tumor grade according to the WHO 1973 (G1-G3) and the recent two-tiered WHO grading system (low grade vs high grade).

In total, $72 / 190$ (38\%) upper urinary tract UCs showed lymph vessel (L1) and/or blood vessel (V1) invasion (Table 1). The presence of vascular invasion was associated with high tumor classification (17/110 (15\%) pT1/pT2 vs 55/80 (69\%) pT3/pT4, $P<0.001)$ and high tumor grade $(7 / 84(8 \%)$ LG vs 65/106 (61\%) HG, $P<0.001)$.

\section{Clinical Findings and Survival Analysis}

Follow-up data were available from 182/190 (96\%) patients. After a mean follow-up of 48 months and a median follow-up of 31 months, progressive disease was observed in 71/182 (39\%) patients including 57 patients who died from cancer and 14 patients who currently are alive with metastatic disease. Mean time to progression was 19 months (median 8, range 0-110). Actuarial overall and metastasis-free survival rates were 63 and $56 \%$ at 5 years and 52 and $45 \%$ at 10 years, respectively.

The development of distant metastases was associated with high tumor classification (9/80 (11\%) pT1 vs 62/102 (61\%) pT2-pT4, $P<0.001$; Figure $3 \mathrm{a}$ and $\mathrm{b}$; Table 2). Median metastasis-free survival of patients with pT1 tumors was 110 months, compared with 29 months for patients with pT2-pT4 tumors. Disease progression was also associated with high tumor grade $(23 / 105(21 \%)$ G1-G2 vs $48 / 77$ (62\%) G3, $P<0.001$ or $12 / 81(15 \%)$ LG vs 59/101 (58\%) HG, $P<0.001$; Figure 3c; Table 2). Median metastasis-free survival of patients with low-grade UC was 102 months, compared with 31 months in patients with high-grade UC. Regarding the different tumor locations, comparable data were noted for both pelvic (14\% pT1 vs 63\% pT2-pT4, $P<0.001 ; 22 \%$ G1-G2 vs $63 \%$ G3, $P<0.001 ; 18 \%$ LG vs $63 \% \mathrm{HG}, P<0.001)$ and ureteral $(7 \%$ pT1 vs $54 \%$
pT2-pT4, $P<0.001 ; \quad 21 \% \quad$ G1-G2 $\quad$ vs $\quad 40 \% \quad$ G3, $P=0.04 ; 8 \%$ LG vs $47 \%$ HG, $P<0.001)$ tumors. Comparing the two different grading systems, the recent two-tiered system appeared to be superior with respect to ureteral UCs. Moreover, regarding moderately differentiated (G2) UCs, 12/70 (17\%) tumors reclassified as low-grade cancers following the new system developed metastatic disease compared to $11 / 24(46 \%)$ tumors reclassified as highgrade cancers $(P<0.001$; Figure $3 \mathrm{~d})$, indicating another possible advantage of the new system. Looking at the subcategories of pelvic pT3 UCs, $3 / 13(23 \%)$ patients with tumors showing only microscopic invasion of renal parenchyma developed metastatic disease compared with 34/40 $(85 \%)$ patients with tumors showing macroscopic invasion and/or spread into peripelvic fat $(P<0.001$; Figure 3e). Eight tumors showed continuous tumor growth within collecting duct lumina, however, without penetration of the duct basal membrane. These tumors were not classified as pT3, and no disease progression was observed among them. Finally, disease progression was associated with the presence of vascular invasion $(19 / 114(17 \%)$ L0 and V0 vs 52/68 (76\%) L1 and/or V1, P<0.001; Figure 3f). Median metastasis-free survival of patients with UC with vascular invasion was 15 months, compared with 110 months of patients with UC without vascular invasion. Regarding the different tumor locations, again comparable data were noted for pelvic $(P<0.001)$ and ureteral $(P<0.001)$ tumors (Table 2$)$. The negative influence of vascular invasion on patient outcome was noted in all pT classifications and grades, but was more pronounced in high classification and high-grade cases (Table 3). No significant prognostic influence was observed for tumor location, patient age, and gender. 

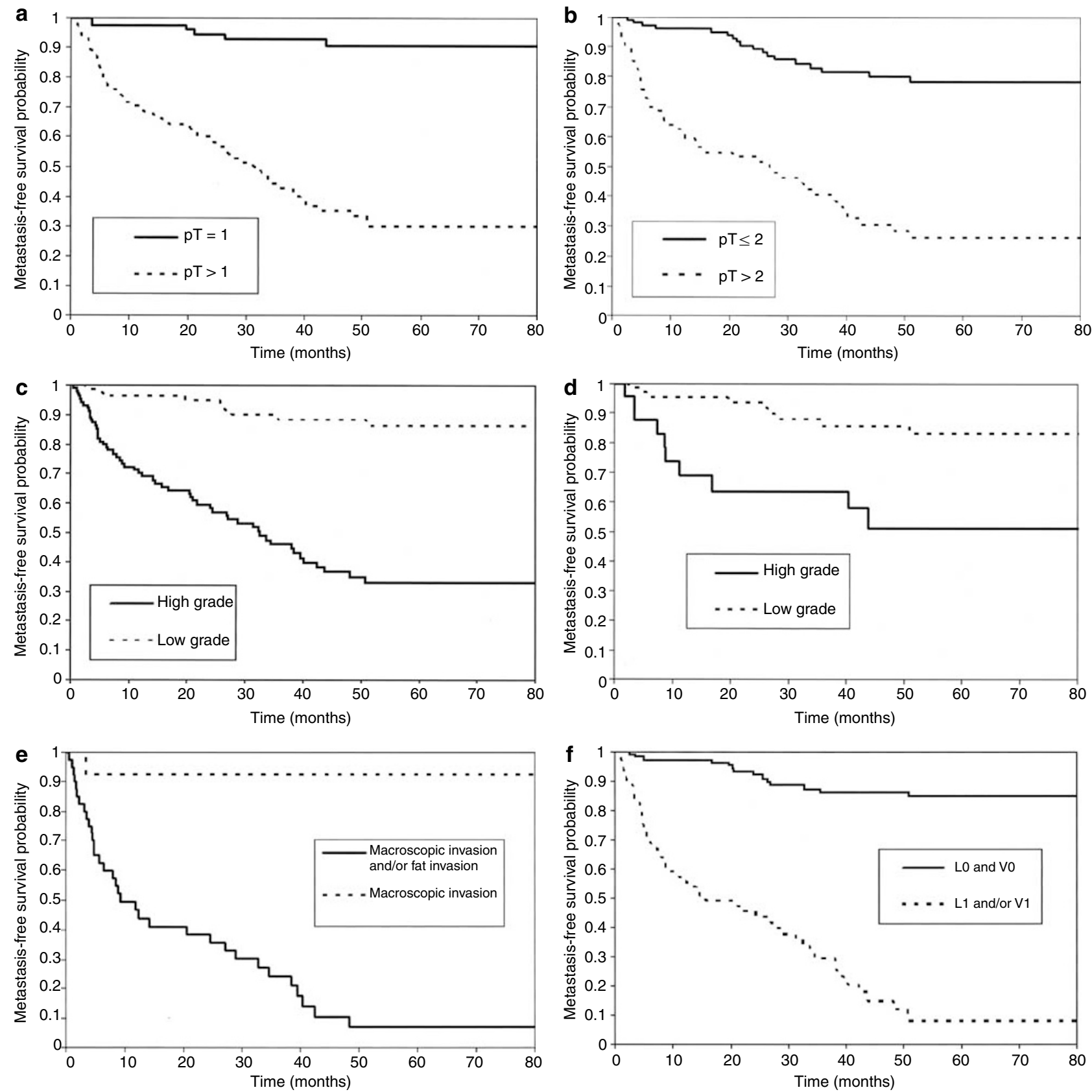

Figure 3 Metastasis-free survival (months) in patients with upper urinary tract urothelial carcinomas related to tumor classification (a pT $=1$ vs pT $>1 ; P<0.001$, Log-Rank test; (b) pT $\leq 2$ vs pT $>2 ; P<0.001$, Log-Rank test) and tumor grade (c high grade vs low grade; $P<0.001$, Log-Rank test). Difference of survival in patients with former moderately differentiated (G2) tumors reclassified according to the recent two-tiered WHO classification (d high grade vs low grade; $P<0.001$, Log-Rank test). Difference of survival in patients with pelvic pT3 tumors related to the amount of renal parenchyma invasion (e microscopic invasion vs macroscopic invasion and/or spread into peripelvic fat, $P<0.001$, Log-Rank test). Metastasis-free survival in patients with upper urinary tract urothelial carcinomas related to presence of vascular invasion (f L0V0 vs L1 and/or V1; $P<0.001$, Log-Rank test).

In multivariate analysis, pT classification $>1$ $(P<0.001$, risk ratio $(\mathrm{RR})=4.4,95 \%$ confidence interval $(\mathrm{CI})=2.0-9.9)$ and vascular invasion $(P<0.001, \quad \mathrm{RR}=5.8, \quad 95 \% \quad \mathrm{CI}=2.9-11.5)$ proved to be independent predictors of metastatic disease. Patients with high-grade tumors according to the two-tiered system were more likely to develop metastatic disease than patients with low-grade tumors, but this difference was not statistically significant $(P=0.06, \quad \mathrm{RR}=2.1, \quad 95 \%$ $\mathrm{CI}=1.0-4.5)$. No independent influence on patient outcome was observed applying the WHO 1973 grading system $(P=0.3, \mathrm{RR}=1.4,95 \% \mathrm{CI}=0.8-2.6$ for $G>2$ ). 
Table 2 Disease progression in upper urinary tract urothelial carcinomas related to tumor classification (pT1-pT4), tumor grade according to WHO 1973 (G1-G3) and the recent two-tiered WHO grading system (low grade vs high grade), as well as vascular invasion

\begin{tabular}{|c|c|c|c|c|c|c|}
\hline & \multicolumn{2}{|c|}{ All tumors $(\mathrm{n}=182)$} & \multicolumn{2}{|c|}{ Pelvic tumors $(\mathrm{n}=128)$} & \multicolumn{2}{|c|}{ Ureteral tumors $(\mathrm{n}=54)$} \\
\hline & $\mathrm{n}$ & $\%$ & $\mathrm{n}$ & $\%$ & $\mathrm{n}$ & $\%$ \\
\hline Overall disease progression & 71 & 39 & 55 & 43 & 16 & 30 \\
\hline pT1 & $9 / 80$ & 11 & $7 / 52$ & 13 & $2 / 28$ & 7 \\
\hline pT2 & $12 / 28$ & 43 & $6 / 16$ & 38 & $6 / 12$ & 50 \\
\hline pT3 & $45 / 67$ & 67 & $37 / 53$ & 70 & $8 / 14$ & 57 \\
\hline pT4 & $5 / 7$ & 71 & $5 / 7$ & 71 & - & - \\
\hline G1 & $0 / 11$ & 0 & $0 / 9$ & 0 & $0 / 2$ & 0 \\
\hline $\mathrm{G} 2$ & $23 / 94$ & 24 & $17 / 67$ & 25 & $6 / 27$ & 22 \\
\hline G3 & $48 / 77$ & 62 & $38 / 52$ & 73 & $10 / 25$ & 40 \\
\hline Low grade & $12 / 81$ & 15 & $10 / 57$ & 18 & $2 / 24$ & 8 \\
\hline High grade & $59 / 101$ & 58 & $45 / 71$ & 63 & $14 / 30$ & 47 \\
\hline L0 and Vo & $19 / 114$ & 17 & $14 / 78$ & 18 & $5 / 36$ & 14 \\
\hline L1 and V0 & $17 / 22$ & 77 & $12 / 15$ & 80 & $5 / 7$ & 71 \\
\hline L0 and V1 & $6 / 12$ & 50 & $5 / 9$ & 56 & $1 / 3$ & 33 \\
\hline L1 and V1 & $29 / 34$ & 85 & $24 / 26$ & 92 & $5 / 8$ & 63 \\
\hline L1 and/or V1 & $52 / 68$ & 76 & $41 / 50$ & 82 & $11 / 18$ & 61 \\
\hline
\end{tabular}

Table 3 Impact of vascular invasion on disease progression related to tumor classification (pT1-pT4) and tumor grade according to the recent two-tiered WHO grading system (low grade vs high grade)

\begin{tabular}{|c|c|c|c|c|c|c|}
\hline & \multicolumn{2}{|c|}{ All tumors $(\mathrm{n}=182)$} & \multicolumn{2}{|c|}{ Pelvic tumors $(\mathrm{n}=128)$} & \multicolumn{2}{|c|}{ Ureteral tumors $(\mathrm{n}=54)$} \\
\hline & $\mathrm{n}$ & $\%$ & $\mathrm{n}$ & $\%$ & $\mathrm{n}$ & $\%$ \\
\hline pT1 L0 V0 & $7 / 70$ & 10 & $6 / 48$ & 13 & $1 / 22$ & 5 \\
\hline PT1 L1 and/or V1 & $2 / 10$ & 20 & $1 / 4$ & 25 & $1 / 6$ & 17 \\
\hline PT2 L0 V0 & $7 / 21$ & 33 & $4 / 12$ & 33 & $3 / 9$ & 33 \\
\hline PT2 L1 and/or V1 & $5 / 7$ & 71 & $2 / 4$ & 50 & $3 / 3$ & 100 \\
\hline PT3 L0 V0 & $5 / 23$ & 22 & $4 / 18$ & 22 & $1 / 5$ & 20 \\
\hline PT3 L1 and/or V1 & $40 / 44$ & 91 & $33 / 35$ & 94 & $7 / 9$ & 78 \\
\hline PT4 L0 V0 & - & - & - & - & - & - \\
\hline PT4 L1 and/or V1 & $5 / 7$ & 71 & $5 / 7$ & 71 & - & - \\
\hline Low-grade L0 Vo & $10 / 74$ & 14 & $8 / 52$ & 15 & $2 / 22$ & 9 \\
\hline Low-grade L1 and/or V1 & $2 / 7$ & 29 & $2 / 5$ & 40 & $0 / 2$ & 0 \\
\hline High-grade L0 V0 & $9 / 40$ & 23 & $6 / 26$ & 23 & $3 / 14$ & 21 \\
\hline High-grade L1 and/or V1 & $50 / 61$ & 82 & $39 / 45$ & 87 & $11 / 16$ & 69 \\
\hline
\end{tabular}

\section{Discussion}

According to literature data, nodal and/or distant metastases occur in $19-54 \%$ of upper tract cancers. $^{2,8,11,12,14-18,20-25,29,32-34}$ Thus, the identification of independent prognostic variables is an essential step to predict disease evolution, thus aiding surveillance strategies, and also to identify patient subgroups that might benefit from adjuvant cancer therapy. Our study is by far the largest systematic investigation of the impact of pT classification, tumor grade, as well as vascular invasion in patients with both pelvic and ureteral UCs with respect to metastasis-free survival.

Outcome of patients in our series was significantly associated with pT classification, thus confirming investigations by others. . $^{5,8,10-13,15-17,20,22,24,25}$ Regarding pT3 pelvic cancer, our data demonstrating significantly poorer outcome of patients with UCs showing macroscopic parenchyma and/or fat invasion compared with patients with UCs showing microscopic parenchyma invasion only are in line with two previous studies. ${ }^{35,36}$ They reported that pT3 UCs with extensive parenchymal invasion (defined as invasion deeper than $5 \mathrm{~mm}$ ) had a prognosis similar to that of pT4 tumors, whereas pT3 UCs without extensive parenchymal invasion (including cases with intraductal spread only) had a prognosis similar to that of pT1/pT2 disease. In their investigation, fat invasion lacked prognostic significance. In another investigation, however, Komatsu et $a l^{37}$ noted that none of six patients with pT3 UCs showing only renal parenchyma invasion died of the disease and that their prognosis was significantly better than that of 11 patients with pT3 UCs invading peripelvic and/or periureteral fat. More recently, Olgac et $a l^{22}$ subdivided 39 pT3 pelvic UCs into three groups defined by (i) sinus fat invasion, (ii) renal parenchyma invasion, and (iii) hilar fat invasion. At the last follow-up, all four patients with 
sinus fat invasion were alive (one of them with metastatic disease).

In summary, the current pT3 category of renal pelvic cancers, defined as 'invasion beyond muscularis into peripelvic fat or renal parenchyma' by the UICC, seems to comprise a heterogeneous group of patients. From a practical point of view, this tumor category could be subdivided by prognostic impact (similar to the recent change in the classification of bladder UCs) into two categories, with one group (pT3a) showing renal parenchyma invasion on a histological level only and the other (pT3b) revealing parenchyma invasion already at gross inspection of the resection specimen and/or showing tumor spread into peripelvic fat. Mere intraductal involvement lacked prognostic significance in the analysis of Yoshimura et $a l^{36}$ and in our series. In consequence, mere intraductal involvement could be excluded from pT3 disease.

Tumor grade was associated with prognosis in univariate analysis, but lacked independent influence on patient outcome with respect to metastasisfree survival in our series. Similar observations have been made in other studies, ${ }^{8,11,12,15-17,22-24}$ whereas only one investigation has identified tumor grade as an independent predictor of overall survival, but not of cancer-specific survival in patients with upper tract cancer. ${ }^{18}$ The new two-tiered grading system, however, has only twice before been applied with respect to prognostic impact in upper urinary tract tumors. ${ }^{22,24}$ In our study, the reclassification of moderately differentiated (G2) tumors into either low- or high-grade cancers proved to be feasible, revealing statistically significant poorer outcome of former G2 cancers that were reclassified as highgrade cancers compared with former G2 that were reclassified as low-grade cancers. Moreover, in the multivariate analysis, the two-tiered system only just missed statistical significance indicating another possible advantage of the new system. It should, however, be noted that no former welldifferentiated (G1) UC progressed, whereas nearly $15 \%$ of low-grade cancers according to the twotiered system developed metastatic disease. This finding indicates that the possible advantage in identifying high-risk patients using the new system is paralleled by a loss of sensitivity with respect to the identification of low or no risk patients. Further studies including bladder cancer cases are needed to clarify this issue.

Angioinvasion was detected in 38\% of cases in our series. It was associated with high tumor stage and grade, and proved to be the strongest predictor of poor patient outcome. Comparable observations have been made by other groups detecting vascular invasion in $25-47 \%$ of cases with prognostic impact both in univariate ${ }^{14,17,22,38}$ and multivariate en $^{23,24,29}$ analyses. Owing to the large sample size, we were able to demonstrate that the prognostic impact of vascular invasion increases with increasing pT classification and grade, as has recently been reported for bladder cancer. ${ }^{39}$ Thus, in our series, the presence of lymph and/or blood vessel invasion doubled the progression rate in pT1-pT2 and/or low-grade cancers, while it quadrupled the progression rate in $\mathrm{pT} 3$ and/or high-grade cancers. More than $90 \%$ of pT3 UCs and more than $80 \%$ of highgrade UCs showing vascular invasion developed metastatic disease, rendering affected patients as ideal candidates for systemic adjuvant cancer therapy.

In conclusion, high pT classification and presence of vascular invasion proved to be the only independent prognostic factors with respect to metastasisfree survival in patients with upper urinary tract UC. The negative influence of vascular invasion on patient outcome was strikingly strong in high pT classification and high-grade cancers. Presence (or absence) of vascular invasion should be commented on separately in the pathology report. The recent two-tiered grading system only just missed independent influence on patient outcome. It may be superior to the WHO 1973 system with respect to the identification of patients at high risk for failure. However, further studies including bladder cancer cases are necessary to clarify this issue.

\section{Acknowledgements}

We thank $\mathrm{Mr} \mathrm{R}$ Christof and Ms A Kaps for photographic expertise and excellent technical support.

\section{References}

1 Genega EM, Porter CR. Urothelial neoplasms of the kidney and ureter. An epidemiologic, pathologic, and clinical review. Am J Clin Pathol 2002;117(Suppl): S36-S48.

2 Huben RP, Mounzer AM, Murphy GP. Tumor grade and stage as prognostic variables in upper urinary tract urothelial tumors. Cancer 1988;62:2016-2020.

3 Das AK, Carson CC, Bolick D, et al. Primary carcinoma of the upper urinary tract. Effect of primary and secondary therapy on survival. Cancer 1990;66:19191923.

4 Charbit L, Gendreau MC, Mee S, et al. Tumors of the upper urinary tract: 10 years of experience. J Urol 1991; 146:1243-1246.

5 Corrado F, Ferri C, Mannini D, et al. Transitional cell carcinoma of the upper urinary tract: evaluation of prognostic factors by histopathology and flow cytometric analysis. J Urol 1991;145:1159-1163.

6 Krogh J, Kvist E, Rye B. Transitional cell carcinoma of the upper urinary tract: prognostic variables and post-operative recurrences. Br J Urol 1991;67: 23-36.

7 Guinan P, Vogelzang NJ, Randazzo R, et al. Renal pelvic cancer: a review of 611 patients treated in Illinois 1975-1985. Cancer Incidence and End Results Committee. Urology 1992;40:293-399. 
8 Cozad SC, Smalley SR, Austenfeld M, et al. Transitional cell carcinoma of the renal pelvis or ureter: patterns of failure. Urology 1995;46:796-800.

9 Lee SH, Lin JS, Tzai TS, et al. Prognostic factors of primary transitional cell carcinoma of the upper urinary tract. Eur Urol 1996;29:266-270.

10 Cheng HL, Chow NH, Tzai TS, et al. Prognostic significance of proliferating cell nuclear antigenexpression in transitional cell carcinoma of the upper urinary tract. Anticancer Res 1997;17:2789-2793.

11 Rey A, Lara PC, Redondo E, et al. Overexpression of p53 in transitional cell carcinoma of the renal pelvis and ureter. Relation to tumor proliferation and survival. Cancer 1997;79:2178-2185.

12 Hall MC, Womack S, Sagalowsky AI, et al. Prognostic factors, recurrence, and survival in transitional cell carcinoma of the upper urinary tract: a 30-year experience in 252 patients. Urology 1998;52:594-601.

13 Jinza S, Takano Y, Iki M, et al. Prognostic significance of p53 protein overexpression in transitional cell carcinoma of the renal pelvis and ureter. Urol Int 1998;60:147-151.

14 Miyake H, Hara I, Gohji K, et al. The significance of lymphadenectomy in transitional cell carcinoma of the upper urinary tract. Br J Urol 1998;82:494-498.

15 Rey A, Lara PC, Redondo E, et al. KI67 proliferation index in tumors of the upper urinary tract as related to established prognostic factors and long-term survival. Arch Esp Urol 1998;51:204-210.

16 Ozsahin M, Zouhair A, Villa S, et al. Prognostic factors in urothelial renal pelvis and ureter tumours: a multicentre Rare Cancer Network study. Eur J Cancer 1999;35:738-743.

17 Morioka M, Jo Y, Furukawa Y, et al. Prognostic factors for survival and bladder recurrence in transitional cell carcinoma of the upper urinary tract. Int J Urol 2001;8: 366-373.

18 Inoue K, Kamada M, Slaton JW, et al. The prognostic value of angiogenesis and metastasis-related genes for progression of transitional cell carcinoma of the renal pelvis and ureter. Clin Cancer Res 2002;8:1863-1870.

19 Fiori E, Cavallaro G, Paparelli C, et al. Significance of T stadium and grading as prognostic factors in transitional cell carcinoma of the ureter. Anticancer Res 2004;24:1921-1924.

20 Mullerad M, Russo P, Golijanin D, et al. Bladder cancer as a prognostic factor for upper tract transitional cell carcinoma. J Urol 2004;172:2177-2181.

21 Park S, Hong B, Kim CS, et al. The impact of tumor location on prognosis of transitional cell carcinoma of the upper urinary tract. J Urol 2004;171:621-625.

22 Olgac S, Mazumdar M, Dalbagni G, et al. Urothelial carcinoma of the renal pelvis. A clinicopathologic study of 130 cases. Am J Surg Pathol 2004;28: 1545-1552.

23 Hong B, Park S, Hong JH, et al. Prognostic value of lymphovascular invasion in transitional cell carcinoma of upper urinary tract. Urology 2005;65:692-696.

24 Holmäng S, Johansson SL. Urothelial carcinoma of the upper urinary tract: comparison between the WHO/ ISUP 1998 consensus classification and WHO 1999 classification system. Urology 2005;66:274-278.
25 van der Poel HG, Antonini N, van Tinteren $\mathrm{H}$, et al. Upper urinary tract cancer: location is correlated with prognosis. Eur Urol 2005;48:438-444.

26 Bol MG, Baak JP, Buhr-Wildhagen S, et al. Reproducibility and prognostic variability of grade and lamina propria invasion in stages Ta, T1 urothelial carcinoma of the bladder. J Urol 2003;169:1291-1294.

27 Lopez-Beltran A, Sauter G, Gasser T, et al. Tumours of the urinary system. Infiltrating urothelial carcinoma In: Eble JN, Sauter G, Epstein JI, Sesterhenn IA (eds) World Health Organization Classification of Tumours. Pathology and Genetics. Tumours of the Urinary System and Male Genital Organs. IARC Press: Lyon, 2004, pp 93-109.

28 Epstein JI, Amin MB, Reuter VR, et al. The World Health Organization/International Society of Urological Pathology consensus classification of urothelial (transitional cell) neoplasms of the urinary bladder. Am J Surg Pathol 1998;22:1425-1448.

29 Hasui Y, Nishi S, Kitada S, et al. The prognostic significance of vascular invasion in upper urinary tract transitional cell carcinoma. J Urol 1992;148: 1783-1785.

30 Sobin LH, Wittekind C, (eds). TNM classification of Malignant Tumors, 6th edn. Wiley-Liss Inc.: New York, 2002.

31 Mostofi FK, Sobin LH, Torloni H, (eds). World Health Organization, International Histological Classification of Tumours No. 10. Histological Typing of Urinary Bladder Tumours, 1973.

32 Racioppi M, D’Addessi A, Alcini A, et al. Clinical review of 100 consecutive surgically treated patients with upper urinary tract transitional tumours. Br J Urol 1997;80:707-711.

33 Nakanishi K, Kawai T, Aida S, et al. Expression of p27(Kip1) protein in transitional cell carcinoma of the upper urinary tract. Mod Pathol 2001;14:371-376.

34 Nakanishi K, Tominaga S, Hiroi S, et al. Expression of survivin does not predict survival in patients with transitional cell carcinoma of the upper urinary tract. Virchows Arch 2002;441:559-563.

35 Fujimoto H, Tobisu K, Sakamoto M, et al. Intraductal tumor involvement and renal parenchymal invasion of transitional cell carcinoma in the renal pelvis. J Urol 1995;153:57-60.

36 Yoshimura K, Arai Y, Fujimoto $\mathrm{H}$, et al. Prognostic impact of extensive parenchymal invasion pattern in pT3 renal pelvic transitional cell carcinoma. Cancer 2002;94:3150-3156.

37 Komatsu H, Tanabe N, Kubodera S, et al. The role of lymphadenectomy in the treatment of transitional cell carcinoma of the upper urinary tract. J Urol 1997;157: 1622-1624.

38 Genega EM, Kapali M, Torres-Quinones M, et al. Impact of the 1998 World Health Organization/International Society of Urological Pathology classification system for urothelial neoplasms of the kidney. Mod Pathol 2005;18:11-18.

39 Quek ML, Stein JP, Nichols PW, et al. Prognostic significance of lymphovascular invasion of bladder cancer treated with radical cystectomy. J Urol 2005; 174:103-106. 